\title{
OS ÓLEOS ESSENCIAIS DE CITRUS TEM AÇÃO INSETICIDA SOBRE A LAGARTA DO MORANGO?
}

\author{
ATAIDE, Julielson Oliveira ${ }^{1}$ \\ FRAGOSO, Débora Ferreira $\mathrm{Melo}^{2}$ \\ PRATISSOLI, Dirceu ${ }^{3}$ \\ PINHEIRO, Patricia Fontes ${ }^{3}$
}

RESUMO: O presente trabalho teve por objetivo avaliar a toxicidade dos óleos essenciais de Citrus limon, Citrus aurantium dulce, Citrus aurantium amara e do D-limoneno frente aos embriões e lagartas de $1^{\circ}$ e $2^{\circ}$ ínstar de Duponchelia fovealis Zeller, 1847 (Lepidoptera: Crambidae). Para os testes biológicos utilizou-se um aerógrafo para pulverizar sobre os insetos as soluções dos óleos essenciais e D-limoneno na concentração de $2 \%$ ( $\mathrm{m} / \mathrm{v}$ ) e o método de imersão para avaliar a mortalidade dos embriões. A toxicidade observada após 72 horas dos óleos essenciais de $C$. Limon e C. a. dulce sobre os embriões foi de 100,00\% e D-limoneno e Citrus aurantium amara de 37,14\% e 37,11\%, respectivamente. Nas lagartas de $1^{\circ}$ ínstar, C. Limon e $C$. a. dulce apresentou 53,75 e 52,50\%, o D-limoneno e Citrus aurantium amara $14,00,13,00 \%$, no $2^{\circ}$ instar $C$. Limon e C. a. dulce apresentou 20,00\%. Os valores estimados de $\mathrm{CL}_{50} \mathrm{CL}_{90}$ de $C$. Limon e $C$. a. dulce sobre os embriões foram, 0,40\%, 0,70\% e 0,13\%, 0,33\%, respectivamente. Pelos resultados obtidos, pode-se inferir que os óleos essenciais de Citrus Limon, Citrus aurantium dulce, Citrus aurantium amara e componente majoritário D-limoneno, apresentaram toxicidade contra $D$. fovealis.

Palavras-Chave: Limão Siciliano. Laranja doce. Laranja amarga. Componentes voláteis.

\section{OS ÓLEOS ESSENCIAIS DE CITRUS TEM AÇÃO INSETICIDA SOBRE A LAGARTA DO MORANGO?}

\begin{abstract}
SUMMARY: This study aimed to evaluate the toxicity of essential oils of Citrus limon, Citrus aurantium dulce, Citrus aurantium amara and D-limonene front of embryos, first instar caterpillars and second instar Duponchelia fovealis Zeller, 1847 (Lepidoptera: Crambidae). For biological tests used an airbrush to spray on insects solutions of essential oils and D-limonene in the concentration of $2 \%(\mathrm{~m} / \mathrm{v})$ and the immersion method to evaluate the mortality of the embryos. The toxicity observed after 72 hours of essential oils of $C$. Limon and $C$. dulce to on embryos was $100.00 \%$ and D-limonene and Citrus aurantium amara of $37.14 \%$ and $37.11 \%$, respectively. In the caterpillars of $1^{\text {st }}$ instar, C. Limon and C. a. dulce presented 53.75 and $52.50 \%$, D-limonene and citrus aurantium amara $14.00,13.00 \%$ in the $2^{\text {nd }}$ instar and C. Limon and C. dulce presented $20.00 \%$. The estimated $\mathrm{LC}_{50} \mathrm{CL}_{90}$ values of $C$. limon and C. a. sweet on the embryos were, $0.40 \%, 0.70 \%$ and $0.13 \%, 0.33 \%$, respectively. From the results, it can be inferred that the essential oils of Citrus limon, Citrus aurantium dulce, Citrus aurantium amara major component and Dlimon $^{1}$ ene, showed toxicity against $D$. fovealis.
\end{abstract}

Keywords: Sicilian lemon. Sweet orange. Bitter orange. Volatile components

\section{INTRODUÇÃO}

O morango (Fragaria x ananassa Duch.) é uma olerícola pertencente à família Rosaceae, que se destaca pelo aspecto atraente e sabor agradável (DAROLT, 2008). Dentre as pequenas frutas

\footnotetext{
${ }^{1}$ Doutorando no Programa de Pós-graduação em Produção Vegetal pela Universidade Federal do Espírito Santo

${ }^{2}$ Pós-Doutoranda no Programa de Pós-graduação em Produção Vegetal pela Universidade Federal do Espírito Santo

${ }^{3}$ Universidade Federal do Espírito Santo
} 
comercializadas é a que possui a maior expressão econômica (OLIVEIRA; SCIVITTARO, 2009). Entretanto alguns insetos pragas são agentes nocivos ao cultivo do morangueiro, prejudicando a produção.

A lagarta do morangueiro Duponchelia fovealis Zeller, 1847 (Lepidoptera: Crambidae) foi identificada no Brasil em 2007 no estado do Paraná na cultura de morangueiro (ZAWADNEAK et al., 2011). Trata-se de um inseto holometábolo, com ciclo médio (ovo-adulto) em torno de 47 dias em temperatura de $20{ }^{\circ} \mathrm{C}$, possuindo 4 ínstares (GILL, 2013). É um inseto de importância, pois atualmente foi relatada atacando 43 famílias e 68 espécies de plantas como hospedeiras (ZAWADNEAK et al., 2011, SOUZA et al., 2013). As lagartas alimentam-se de folhas novas, brotos, flores, frutos e restos vegetais em decomposição (Brambila \& Stocks, 2010).

Nos países de origem da lagarta usam-se os ingredientes ativos lambda-cialotrina, deltametrina e spinosade para o controle de D. fovealis (FRANCO; BAPTISTA, 2010). No Brasil, por não ter um inseticida sintético para $D$. fovealis, o seu controle químico é feito com base em outras pragas, como as traça-do-tomateiro, Tuta absoluta (Meyrick, 1917) (Lepidoptera: Gelechiidae), utilizando o ingrediente ativo clorfenapir (SOUZA et al., 2013).

O método de controle químico tem contribuído para o aumento da produção agrícola, entretanto, o uso incorreto e indiscriminado durante várias décadas levou à acumulação de resíduos tóxicos em alimentos, contaminação da água e do solo, intoxicação de produtores rurais, seleção de pragas resistentes, entre muitos outros problemas (CORRÊIA; SALGADO, 2011), o uso de produtos naturais para o controle desse inseto-praga pode ser uma alternativa viável (BRAHMI et al., 2016). Nesse sentido, os óleos essenciais de plantas que são constituintes voláteis de plantas, apresentam potencial inseticida podem ser viáveis no manejo de $D$. fovealis.

Os óleos essenciais para o controle de doenças de plantas e insetos têm sido relatados como uma alternativa promissora para o manejo integrado de pragas e doenças (TRIPATHI et al., 2009), atrelado a algumas vantagens, como menor desenvolvimento de resistência dos insetos aos inseticidas pelo complexo de princípios ativos presentes nas plantas; baixa toxicidade a outros insetos úteis (polinizadores e inimigos naturais de insetos pragas), aves, peixes e mamíferos, incluindo o ser humano (ISMAN, 2000).

Dentre estes, os óleos de Citrus, podem ser promissores para o controle da lagarta do morangueiro, uma vez que possui atividade inseticida em diversos insetos-praga, como Callosobruchus maculatus (Fabricius, 1775) (Coleoptera: Bruchidae), Ceratitis capitata (Wiedemann, 1824) (Diptera: Tephritidae) (DUTRA et al., 2016, RUIZ et al., 2014).

Dessa forma, o objetivo desse trabalho foi averiguar se existe ação inseticida dos óleos essenciais de Citrus sobre a lagarta do morango.

\section{MATERIAL E MÉTODO}

Os óleos essenciais de limão siciliano Citrus limon, laranja doce Citrus aurantium dulce, laranja amarga Citrus aurantium amara e o componente majoritário D-limoneno foram adquiridos da empresa Ferquimica Indústria e Comércio Ltda. (Vargem Grande, São Paulo, Brasil), extraídos em escala industrial por hidrodestilação e arraste de vapor de água (DAPKEVICIUS et al., 1998).

Os insetos utilizados no experimento foram retirados da criação estoque do setor de entomologia do Núcleo de Desenvolvimento Científico e Tecnológico em Manejo Fitossanitário (NUDEMAFI) do Centro de Ciências Agrárias e Engenharias (CCAE) da Universidade Federal do Espírito Santo (UFES), provenientes inicialmente da região serrana do Estado do Espírito Santo, do município de Santa Maria de Jetibá $\left(20^{\circ} 2^{\prime} 27^{\prime \prime} \mathrm{S}, 40^{\circ} 44^{\prime} 45^{\prime \prime} \mathrm{W}\right)$. 
Os insetos foram mantidos em salas climatizadas, sob condições de $25 \pm 1{ }^{\circ} \mathrm{C}, 70 \pm 10 \%$ umidade relativa e 14 horas de fotofase. Adultos recém-emergidos foram transferidos para gaiolas $(20 \times 20 \mathrm{~cm})$ confeccionadas com tubo do tipo PVC, forrado internamente com papel sulfite e fechadas na base inferior com isopor, também revestido com papel sulfite. A extremidade da gaiola foi fechada com tecido do tipo voil para evitar a fuga dos insetos. Mel em solução a $10 \%$ embebido em algodão foi oferecido como alimento aos adultos. As posturas foram recolhidas diariamente por meio da troca do papel que revestia as gaiolas.

Os papéis contendo os ovos foram tratados através da imersão por 10 segundos em solução de formaldeído em $1 \%$ (v/v) e posteriormente em solução de sulfato de cobre em 17\%; procedimentos esses que ocorreram dentro de bancada de fluxo laminar. Após a secagem, foram transferidos para tubos de vidro de fundo chato $(8,5 \times 2,5 \mathrm{~cm})$ contendo dieta artificial proposta por Kinge Hartley (1985) modificada.

Os óleos essenciais de Citrus foram diluídos a $2 \%(\mathrm{~m} / \mathrm{v})$ usando acetona $2 \%(\mathrm{~m} / \mathrm{v})$, Tween 80 0,05\% (m/v) e água (Chagas Filho et al., 2005). Como testemunha (controle negativo) utilizou-se a mistura isenta de óleo essencial, para o controle positivo foi utilizado Clorfenapir na dosagem comercial $100 \mathrm{~mL} / 100 \mathrm{~L}$.

O experimento desenvolveu-se em 3 bioensaios, sendo o primeiro a ação do óleo essencial sobre os embriões e o segundo sobre lagartas de $1^{\circ}$ ínstar e terceiro sobre lagartas de $2^{\circ}$ ínstar. No primeiro bioensaio, foram contabilizados grupos de 20 ovos de 48 horas, em pré-formação de embriões, por repetição, recortados do papel sulfite da criação estoque. Posteriormente, foram imersos no tratamento por 30 segundos e dispostos em placas de Petri $(9,0 \times 1,5 \mathrm{~cm})$, revestidas com papel filtro, tampadas e armazenas em câmaras climatizadas nas condições citadas anteriormente. Cada placa correspondeu a uma repetição, totalizando 10 repetições.

A avaliação foi feita diariamente até 72 horas após a imersão, onde foi verificada a mortalidade dos embriões através do número de lagartas não eclodidas por repetição.

No segundo e no terceiro bioensaio, foi realizada a avaliação sobre o $1^{\circ}$ e $2^{\circ}$ ínstar das lagartas de D. fovealis, através da pulverização com o auxílio de um aerógrafo com pressão de $15 \mathrm{lb} \mathrm{pol}^{2}$, contendo 2 $\mathrm{mL}$ de solução, sobre placas de Petri $(9,0 \times 1,5 \mathrm{~cm})$ revestidas com papel filtro com 20 lagartas e um disco de dieta artificial $(1,5 \mathrm{~cm}$ diâmetro x 0,5 cm altura). Em seguida, as placas foram tampadas, vedadas com plástico filme e mantidas em câmeras climatizadas (metodologia adaptada de SILVA, 2014).

Para estes bioensaios, cada placa de Petri correspondeu a uma repetição, obtendo 5 repetições para cada tratamento ( $1^{\circ}$ e $2^{\circ}$ ínstar).

As avaliações foram feitas 72 horas após a aplicação, através do toque do pincel de numeração 0,1 nas lagartas para verificar a mortalidade dos indivíduos.

Foi utilizado o delineamento experimental inteiramente casualizado. Os resultados foram testados para normalidade e homogeneidade de variância, segundo os testes de Shapiro-Wilk e Bartlett ao nível de $5 \%$ probabilidade, respectivamente. Posteriormente, os dados foram submetidos à análise de variância (ANOVA) e as médias comparadas pelo teste de Tukey a $5 \%$ de probabilidade por meio do programa Sas 9.0 (SAS INSTITUTE, 2002).

A estimativa da concentração letal do óleo essencial de Citrus limon, Citrus aurantium dulce foi feita sobre embriões. Para isso, foram utilizadas 7 concentrações dos óleos essenciais de limão siciliano e laranja doce espaçadas em escala logarítmica, sendo o limite inferior (concentração que causa a morte de cerca de $10 \%$ dos insetos) e superior (concentração que causa a morte de cerca de $90 \%$ dos insetos) determinados mediante ensaios preliminares. Na testemunha foi utilizada acetona a $2 \%(\mathrm{~m} / \mathrm{v})$ e Tween 80 a $0,05 \%(\mathrm{~m} / \mathrm{v})$ diluídos em água destilada (CHAGAS FILHO et al., 2005). Esta etapa do bioensaio foi 
realizada conforme os procedimentos adotados no pimeiro ensaio da etapa anterior. A mortalidade dos embriões foi avaliada até 72 horas. Foi utilizado o delineamento experimental inteiramente casualizado (DIC) com 4 repetições contendo 20 ovos de 48 horas em pré-formação de embrião, em cada repetição. A concentração letal foi estimada usando a análise de Probit por meio do programa Sas 9.0 (SAS INSTITUTE, 2002).

\section{RESULTADO E DISCUSSÃO}

Os resultados obtidos seguiram distribuição normal e homogeneidade de variância ( $\mathrm{P}>0,05)$. Os óleos essenciais de limão siciliano e laranja doce causou mortalidade em todos os tratamentos, entretanto laranja amarga e o D-limoneno só apresentaram mortalidade em embriões e lagartas de $1^{\circ}$ ínstar. Contudo os óleos essenciais de limão siciliano e laranja doce, em embriões $\left(\mathrm{F}_{5,41}=4150,2 ; \mathrm{P}<0,001\right)$, mortalidade de $100 \%$, não diferindo do inseticida clorfenapir. Em lagartas foi observada baixa mortalidade, 53,75 e $52,50 \%\left(\mathrm{~F}_{5,22}=659,5 ; \mathrm{P}<0,001\right)$ de $1^{\circ}$ ínstar e 20,00\% $\left(\mathrm{F}_{3,16}=495 ; \mathrm{P}<0,001\right) 2^{\circ}$ ínstar respectivamente. $\mathrm{O}$ componente majoritário D-limoneno e o óleo essencial de laranja amarga obtiveram baixa mortalidade de 37,14 e $37,11 \%$ em embriões $\left(F_{5,41}=4150,2 ; \mathrm{P}<0,001\right), 14,00 \%$ e $13,00 \%$ em lagartas $1^{\circ}$ ínstar $\left(F_{5}\right.$, $\left.{ }_{22}=659,5 ; \mathrm{P}<0,001\right)$ respectivamente (Tabela 1$)$.

Tabela 1. Taxa de mortalidade (\%) de embrião e lagartas de $1^{\circ}$ e $2^{\circ}$ ínstar de Duponchelia fovealis causada pelos óleos essenciais de Citrus limon, Citrus aurantium dulce, Citrus aurantium amara e seu componente majoritário D-limoneno a $2 \%(\mathrm{~m} / \mathrm{v})$, após 72 horas. Temperatura de $25 \pm 1^{\circ} \mathrm{C}$, umidade relativa de $70 \pm 10 \%$ e fotofase de 12 horas.

\begin{tabular}{cccc}
\hline \multicolumn{4}{c}{ Mortalidade (\%) } \\
\hline \multirow{2}{*}{ Tratamentos } & \multicolumn{3}{c}{ Fase do inseto } \\
\cline { 2 - 4 } & $\mathbf{1}^{\mathbf{0}}$ Bioensaio & $\mathbf{2}^{\mathbf{0}}$ Bioensaio & $\mathbf{3}^{\mathbf{0}}$ Bioensaio \\
\hline C. limon & $100 \pm 0,00 \mathrm{a}$ & $53,75 \pm 1,25 \mathrm{~b}$ & $20,00 \pm 3,16 \mathrm{~b}$ \\
C. a. dulce & $100 \pm 0,00 \mathrm{a}$ & $52,50 \pm 1,44 \mathrm{~b}$ & $20,00 \pm 2,23 \mathrm{~b}$ \\
D-limoneno & $37,14 \pm 1,24 \mathrm{~b}$ & $14,00 \pm 1,87 \mathrm{c}$ & $0,00 \pm 0,00 \mathrm{c}$ \\
C. a. amara & $37,11 \pm 1,24 \mathrm{~b}$ & $13,00 \pm 2,00 \mathrm{c}$ & $0,00 \pm 0,00 \mathrm{c}$ \\
Clorfenapir & $100 \pm 0,00 \mathrm{a}$ & $100 \pm 0,00 \mathrm{a}$ & $100 \pm 0,00 \mathrm{a}$ \\
Testemunha & $3,00 \pm 0,00 \mathrm{c}$ & $2,00 \pm 1,22 \mathrm{c}$ & $5,00 \pm 0,00 \mathrm{c}$ \\
\hline P-valor & 2,66 & 3,46 & 5,53 \\
\hline
\end{tabular}

Médias ( \pm EP) seguidas pela mesma letra, na coluna, não diferem estatisticamente entre si pelo teste de Tukey, a 5\% de probabilidade.

O D-limoneno componente majoritário de ambos os óleos essenciais obteve baixa mortalidade sobre as lagartas de $1^{\circ}$ ínstar de D. fovealis (14\%). Isso demonstra que isoladamente o componente majoritário causou baixo efeito sobre essas lagartas e depende de outro componente presente nos óleos essenciais citados que atuem em sinergismo para causar maior mortalidade. O mesmo componente majoritário foi avaliado em larvas de Aedes albopictus (Skuse, 1894) (Diptera: Culicidae) obteve uma $\mathrm{CL}_{50} 0,004175 \%$ (m/v) (LIU et al., 2015).

O mesmo efeito ocorreu no trabalho desenvolvido por Shukla et al. (2011), em que os óleos essenciais de falsa melissa Lippia alba (Verbenaceae) e escova-de-garrafa Callistemon lanceolatus (Myrtaceae) e o seu componente majoritário geranial, foram avaliado sobre larvas de Callosobruchus chinensis (Linnaeus, 1758) (Coleoptera: Chrysomelidae), obtendo maiores mortalidades atreladas aos óleos essenciais quando comparado ao seu componente majoritário, sendo para L. Alba de 22,81 a 
$50.93 \%$ de mortalidade, $C$. lanceolatus 42.81 a $77.18 \%$ e para geranial 10.31 a $41.56 \%$.

A baixa mortalidade verificada para o óleo essencial de laranja amarga $(13,00 \%)$ pode estar relacionada aos componentes existentes no óleo essencial, que causam diferentes efeitos sobre os insetos (ISMAN, 2000). O mesmo óleo essencial apresentou efeito inseticida sobre mosquito Culex pipiens (Linnaeus, 1758) (Diptera: Culicidae), alcançando uma $\mathrm{CL}_{50} \mathbf{0 , 0 0 3 9 \%}$ (MICHAELAKIS et al., 2009).

Os óleos essenciais extraídos de limão siciliano têm efeitos que consistem em repelência e deterrência (SU, 1976), o que justifica a baixa mortalidade verificada nos testes ingestão e contato 53,75\% para o $1^{\circ}$ ínstar e $20,00 \%$ no $2^{\circ}$ ínstar do presente trabalho. O mesmo óleo essencial foi aplicado em lagartas $3^{\circ}$ ínstar de Thyrinteina arnobia (Stoll, 1782) (Lepidoptera: Geometridae) causando mortalidade inferior a 20,00\% (SOARES et al., 2011).

Para laranja doce a porcentagem de mortalidade $52,50 \%$ para o $1^{\circ}$ ínstar e $20,00 \%$ para lagartas de $2^{\circ}$ ínstar foi semelhante ao limão siciliano, demonstrando assim, baixa atividade quando comparado à mortalidade de outros óleos em outros lepidópteros (NICULAU et al., 2013).

Porém, existem relatos de óleos essenciais que causaram mortalidade satisfatória em insetospraga, como a ação inseticida do óleo essencial de aroeira-salso Schinus molle L. Anacardiaceae sobre Sitophilus zeamais Most. 1855 (Coleoptera: Curculionidae) que obteve uma $\mathrm{Cl}_{50} 0,25 \mu \mathrm{L} / \mathrm{cm}^{2}$ e $\mathrm{Cl}_{90} 1,92$ $\mu 1 / \mathrm{cm}^{2}$ (FERNANDES; FAVERO, 2014), entre outros óleos como o manjericão Ocimumbasilicum $L$. Lamiaceae $\left(\mathrm{Cl}_{50} 17,67 \mu \mathrm{L} / \mathrm{L}\right)$, laranja-azeda Citrus aurantium L. Rutáceae $\left(\mathrm{Cl}_{50} 69,36 \mu \mathrm{L} / \mathrm{L}\right)$, hortelã-verde Mentha spicata L. Lamiaceae $\left(\mathrm{Cl}_{50} 27.51 \mu \mathrm{L} / \mathrm{L}\right)$ e quebra-faca Crotonpulegiodorus Bail Euphorbiaceae $\left(\mathrm{Cl}_{50} 48.66 \mu \mathrm{L} / \mathrm{L}\right)$ sobre Rhyzopertha dominica Fabr. 1972 (Coleoptera: Bostrichidae) (SOUZA et al., 2016).

Na estimativa da concentração letal $\left(\mathrm{CL}_{50}\right.$ e $\left.\mathrm{CL}_{90}\right)$, os dados adequaram-se ao modelo de Probit, mostrando um $x^{2}$ não significativo e baixa heterogeneidade (Tabela 2).

Tabela 2. Inclinação das curvas de concentração-resposta e concentração letal $\left(\mathrm{CL}_{50}\right.$ e $\left.\mathrm{CL}_{90}\right)$ do óleo essencial de Citrus limon, Citrus aurantium dulce, sobre embriãos de Duponchelia fovealis. Temperatura: $25 \pm 1^{\circ} \mathrm{C}$, UR $70 \pm 10 \%$ e de fotofase 12 horas.

\begin{tabular}{|c|c|c|c|c|c|c|c|}
\hline O. essenciais & $\mathbf{G L}^{\mathbf{a}}$ & $\mathbf{N}^{\mathbf{b}}$ & $\begin{array}{l}\text { Inclinação } \\
\text { EPM }^{c}\end{array}$ & $\begin{array}{c}\text { CL }_{50} \\
(\mathbf{I C ~ 9 5 \%})^{d}\end{array}$ & $\begin{array}{c}\text { CL }_{90} \\
(\mathrm{IC} 95 \%)^{\mathrm{d}}\end{array}$ & $\mathrm{X}^{2 \mathrm{e}}$ & P-valor ${ }^{f}$ \\
\hline C. limon & 5 & 700 & $5,19 \pm 0,43$ & $\begin{array}{c}0,40 \\
(0,37-0,42)\end{array}$ & $\begin{array}{c}0,70 \\
(0,65-0,77)\end{array}$ & 2,73 & 0,7404 \\
\hline C. a. dulce & 5 & 700 & $3,18 \pm 0,27$ & $\begin{array}{c}0,13 \\
(0,11-0,14)\end{array}$ & $\begin{array}{c}0,33 \\
(0,30-0,38)\end{array}$ & 5.99 & 0.3068 \\
\hline $\begin{array}{l}\text { a Graus de liberda } \\
{ }^{\mathrm{c}} \text { Inclinação } \pm \text { Err } \\
{ }^{\mathrm{e}} \text { Teste qui-quadr }\end{array}$ & & & \multicolumn{5}{|c|}{$\begin{array}{l}\text { b Número de insetos usados no teste. } \\
\text { d Intervalo de confiança das } \mathrm{CL}_{50} \text { e } \mathrm{CL}_{90} \text { a } 95 \% \text { de probabilidade. } \\
{ }^{\mathrm{d}} \text { Probabilidade de significância }\end{array}$} \\
\hline
\end{tabular}

O óleo essencial de laranja doce apresentou na fase de embrião uma $\mathrm{CL}_{50} 0,13 \%$ e $\mathrm{CL}_{90} 0,33 \%$, contudo o óleo essencial de limão siciliano exibiu uma $\mathrm{CL}_{50} 0,40 \%$ e $\mathrm{CL}_{90} 0,70 \%$, ou seja, o óleo de laranja doce foi mais tóxico. Contudo os inseticidas deltametrina, NeemPro, Natunem ${ }^{\circledR}$, Neemseto® foram testados sobre ovos de Neoleucinodes elegantalis (Guenée, 1854) (Lepidoptera: Crambidae), apresentaram concentrações letais $\left(\mathrm{CL}_{50 \mathrm{~s}}\right)$ entre 0,029 a 4,19\% (FRANÇA, 2013). Entretanto os inseticidas methomil, cartap e abamecti apresentaram entre 40 a $51 \%$ de inviabilidade de ovos de $N$. elegantalis (BARTOLI et al., 2013). Para Spodoptera frugiperda (Smith \& Abbot, 1797) (Lepidoptera: Noctuidae) o inseticida clorfenapir apresentou 25,25\% de inviabilidade dos ovos (SOARES, 2018). 


\section{CONCLUSÃO}

Os óleos essenciais de citrus causa toxicidade em embrião e lagartas de $1^{\circ}$ e $2^{\circ}$ ínstar $D$. fovealis. Entretanto o óleo essencial de laranja amarga e o D-limoneno apresentou baixa toxicidade em embrião e lagartas de $1^{\circ}$ instar.

\section{REFERENCIAS}

BORTOLI, S.A. et al. Ação de inseticidas sobre os ovos e lagartas da broca-pequena-do-fruto do tomate, em bioensaio de laboratório. Arq. Inst. Biol, Jaboticabal, v.80, n.1, p.73-82, jan./mar. 2013.

BRAHMI, F. et al. Chemical composition and in vitro antimicrobial, insecticidal and antioxidant activities of the essential oils of Mentha pulegium L. and Mentha rotundifolia L. Huds growing in Algeria. Industrial Crops and Produc, Bejaia, v. 88, p.96-105, 15, out. 2016.

BRAMBILA, J.; STOCKS, I. The European Pepper Moth, Duponchelia fovealis Zeller (Lepidoptera: Crambidae), a Mediterranean Pest Moth Discovered in Central Florida. Pest Alert created, Florida, p. 14, 2, dez.2010.

CHAGAS FILHO, N.R. et al. Desenvolvimento ninfal de Myzus persicae (Sulzer, 1776) (Hemiptera: Aphididae) sobre berinjela em diferentes temperaturas. Bragantia, Campinas, v.64, n.2, p.257-262. 2005.

CORREAA, J.C.R.; SALGADO, H.R.N. Atividade inseticida das plantas e aplicações: revisão. Rev. Bras. Pl. Med., Botucatu, v.13, n.4, p.500-506, 2011.

DAPKEVICIUS, A. et al. Antioxidant activity of extracts obtained by different isolation procedures from some aromatic herbs grown in Lithuania. Journal Science Food Agriculture, v.77, p.140-146, 23, set.1998.

DAROLT, M.R. Morango orgânico: opção sustentável para produtores, consumidores e meio ambiente. Revista campo e negócio, n.34, p.58-61, 34, mar. 2008.

DUTRA, K. A. et al.. Control of Callosobruchus maculatus (FABR.) (Coleoptera: Chrysomelidae: Bruchinae) in Vigna unguiculata (L.) WALP. With essential oils from four Citrus spp. plants. Journal of Stored Products Research, Recife, v.68, p.25-32, jul. 2016.

FRANÇA, S. M. Manejo de Neoleucinodes elegantalis (Guenée), (Lepidoptera: Crambidae) em tomateiro no agreste do Estado de Pernambuco: estudos comportamentais uso de iscas tóxicas, inseticidas botânicos e sintéticos. 2013. 64p. Tese (Doutorado) - Universidade Federal Rural de Pernambuco. Recife.

FRANCO, M.C.; BAPTISTA, M.C. Duponchelia fovealis Zeller - nova praga no Portugal. Frutas, legumes e flores - a revista dos profissionais, v.110, p.34-35, 2010.

GILL, S. European Pepper Moth Found in Maryland. Integrated Pest Management for Commercial Horticulture. University of Maryland Extension - Solutions in your Community. Pest Alert Created, p.14, 2013.

ISMAN, M.B. Plant essential oils for pest and disease management. Crop Protection, Vancouver, v.19, p.603-8, 12, set.2000.

LIU, X.CH. et al. Larvicidal activity of the essential oil from Tetradium glabrifolium fruits and its constituents against Aedes albopictus. Pest Management Science, Beijing, v.71, p.1582-1586, 14, jan.2015. 
MICHAELAKIS, A. et al. Citrus essential oils and four enantiomeric pinenes against Culex pipiens (Diptera: Culicidae). Parasitology Research, Kifissia Athens, v. 105, p.769-773, 20, abr.2009.

NICULAU,E.S. et al. Atividade Inseticida de Óleos Essenciais de Pelargonium graveolens 1'Herit E Lippia alba (Mill) N. E. Brown sobre Spodoptera frugiperda (J. E. Smith). Química Nova São Paulo, v.36, p.1391-1394, 2, out. 2013.

OLIVEIRA, R. P.; SCIVITTARO, W. B. Produção de frutos de morango em função de diferentes períodos de vernalização das mudas. Horticultura Brasileira, v.27, n.1, p. 91-95, jan./mar.2009.

RUIZ, M.J. et al. Toxic Effect of Citrus Peel Constituents on Anastrepha fraterculus Wiedemann and Ceratitis capitata Wiedemann Immature Stages. Journal of Agricultural and Food Chemistry, v.62, p. 10084-10091, 19, set.2104.

SHUKLA, R. et al. Efficacy of essential oils of Lippia alba (Mill.) N.E. Brown and Callistemon lanceolatus (Sm.) Sweet and their major constituents on mortality, oviposition and feeding behavior of pulse beetle, Callosobruchus chinensis L. Journal of the Science of Food and Agriculture, v.91, p.2277-2283, 5 maio 2011.

SILVA, R. T. L. Efeito de entomopatógenos e extratos vegetais sobre ApismelliferaL. (Hymenoptera: Apidae). 2014. 104p. Dissertação (Mestrado) - Universidade Tecnológica Federal do Paraná, Dois Vizinhos.

SOARES, C. S. A. et al. Ação Inseticida de Óleos Essenciais Sobre a Lagarta Desfolhadora Thyrinteina arnobia (Stoll) (Lepidoptera: Geometridae). Revista Verde de Agroecologia e Desenvolvimento Sustentável, v.6, p.154 -157 abr./jun. 2011.

SOARES, W. S. Ação de diferentes grupos químicos de inseticidas sobre o desenvolvimento embrionário, emergência de Spodoptera frugiperda (Smith \& Abbot, 1797) (LEPIDOPTERA: NOCTUIDAE) e seletividade à vespidaes predadores. 2018. 58p. Dissertação (Mestrado) - Universidade Federal de Uberlândia. Minas Gerais.

SOUZA, V. N. et al. Fumigation Toxicity of Essential Oils Against Rhyzopertha dominica (F.) in Stored Maize Grain. Rev. Caatinga, Serra Talhada, v. 29, n. 2, p. 435 - 440 abr./jun. 2016.

SOUZA, J.C. et al. Ocorrência de nova praga nas lavouras de morango no Sul de Minas. EPAMIG. Circular Técnica, n.180, p.1-5,.2013.

TRIPATHI, A.K. et al. Review on prospects of essential oils as biopesticide in insect-pest management. Journal of Pharmacognosy and Phytotherapy, Jorhat Assam, v.1, n.5, p.52-63, novembro. 2009.

ZAWADNEAK, M.A. et al.. Morango: novo desafio. Cultivar HF, Brasília, p.30-32, 2011. 